\title{
Determinantes en la elección del tipo de combustible para cocción de alimentos en asentamientos
} humanos

\author{
Carlos Meza ${ }^{1}$ y Carlos Narváez² \\ ${ }^{1}$ Miembro de la Red de Expertos Iberoamericanos en Energía y funcionario de la Gerencia Comercial de ALBA GENERACIÓN. \\ Managua, Nicaragua. \\ ${ }^{2}$ Profesor horario, Departamento de Economía Aplicada, Universidad Centroamericana. Rotonda Rubén Darío 150 mts. al oeste. \\ Apartado 69, Managua, Nicaragua. Correo electrónico: carlnarsil@yahoo.com
}

Recibido: enero de 2010 / Aceptado: febrero de 2010

EL ARTÍCULO PRESENTA LOS DETERMINANTES EN LA ELECCIÓN DEL TIPO DE combustible primario para cocción de alimentos en cuatro asentamientos humanos urbanos localizados en los departamentos de Managua y León. Se eligió un modelo logístico binario (Logit) que permitió concluir que los factores que inciden en la elección son: el ingreso, los niveles de educación, el departamento al que pertenecen los barrios, número de miembros del hogar y la edad del cabeza de familia. Este estudio ofrece un punto de inicio en la discusión de los aspectos de relevancia en la demanda de combustibles a nivel urbano en Managua y León.

Palabras clave: Gas Licuado de Petróleo / leña / cocción de alimentos / determinantes / modelo logístico

\section{Introducción}

La elección del tipo de combustible para cocción de alimentos es un tema de gran importancia en el bienestar de las familias, especialmente de las que se encuentran en condiciones de pobreza. Nicaragua -al igual que gran parte de los países en vías de desarrollo- es dependiente de combustibles fósiles y biomasa. Según datos obtenidos en el Censo 2005 (Instituto Nacional de Información de Desarrollo, 2005a), cerca de 6 de cada 10 familias a nivel nacional utilizan leña para cocinar.

El consumo de Gas Licuado de Petróleo (GLP) envasado en cilindros de $11.34 \mathrm{~kg}$ ha venido creciendo a lo largo de los últimos 20 años. Sin embargo, este crecimiento es desigual, puesto que se concentra en la ciudad de Managua y en las cabeceras departamentales.

Existe una serie de determinantes socio-económicos, energéticos y culturales asociados a la elección del combustible primario para cocción de alimentos en el hogar, que deben ser 
examinados y analizados. Es por esta razón que este estudio exploratorio pretende analizar, a través de un enfoque econométrico, los determinantes en la elección del tipo de combustible primario para cocción de alimentos utilizado por familias de cuatro asentamientos humanos de las ciudades de Managua y León.

En la segunda sección de este artículo se presentan los materiales y método empleados para realizar la investigación. En la tercera sección se abordan aspectos geográficos, poblacionales y de extensión territorial de los barrios. En la cuarta, algunas consideraciones en materia socio-económica que permitan contextualizar al lector sobre la situación en que viven los habitantes.

El acápite cinco muestra algunas referencias a trabajos realizados anteriormente en Nicaragua y el resto del mundo, así como los enfoques desde los cuales se ha abordado este tema. El sexto acápite presenta el marco teórico elegido como base para el análisis econométrico y el séptimo, la metodología aplicada para utilizar el modelo logístico binario.

El acápite ocho especifica las variables seleccionadas como explicativas y estadísticas descriptivas de las mismas. En el acápite nueve se discuten los resultados y en el acápite final se plantean las conclusiones.

\section{Materiales y método}

\subsection{Encuesta}

Se utilizaron datos de la encuesta realizada por el Ministerio de Energía y Minas (MEM, 2009) en los meses de mayo y junio de 2009. El objetivo de la misma era recolectar información que permitiera determinar la capacidad de pago por el servicio eléctrico en cuatro asentamientos urbanos con problemas de mora y conexiones ilegales. Inicialmente, el MEM realizó una encuesta piloto en la que se detectaron algunas fallas y se procedió a ajustar el instrumento de captura. Es a partir de la base de datos de la encuesta ajustada y probada que realizamos esta investigación, por considerar que reúne las variables necesarias para el cumplimiento de nuestros propósitos.

La población total es de 2,500 familias. En el departamento de Managua, los barrios seleccionados fueron el Arnoldo Alemán y el 4 de abril, los que cuentan con 1,000 y 800 hogares respectivamente. En el caso del departamento de León, los barrios seleccionados fueron el Lino Argüello y el Mariana Sansón con 400 y 300 hogares respectivamente.

\subsection{Definición del tamaño de la muestra}

Se utilizó la base de datos completa de los cuatro barrios encuestados por considerar que tienen condiciones socio-económicas similares. Le encuesta cuenta con un total de 404 hogares encuestados, de los 2,500 del total de la población, con una representatividad del $16.2 \%$. Es preciso indicar que debido a datos faltantes en algunas variables, al momento de estimar el modelo se trabajó con 388 observaciones. Esta muestra presenta un nivel 
de confiabilidad superior al 95\% usando la fórmula de poblaciones finitas y muestreo completamente aleatorio de Munch Galindo, $1996^{1}$.

\section{Ubicación geográfica, población y extensión territorial de los asentamientos}

El barrio Mariana Sansón se encuentra ubicado en el sector sureste del área sub-urbana de la ciudad de León, cabecera departamental del departamento de León. Cuenta con una población aproximada de 2,897 habitantes en un territorio de 107 manzanas.

El barrio Lino Argüello fue fundado en 2005 por la Alcaldía de León. El origen de su nombre se debe a Lino Argüello (10-agosto-1877/ 15-agosto-1937), poeta. Está ubicado en el departamento de León, en el occidente del país, específicamente en el bypass que conduce a Chinandega, de los laboratorios Divina $2 \mathrm{~km}$ al este y $600 \mathrm{mts}$ al sur. Cuenta con una población aproximada de 1,300 habitantes en una extensión territorial de 16 manzanas.

El barrio 4 de abril se encuentra en el municipio de Mateare, en el departamento de Managua cercano a Ciudad Sandino. Cuenta con una población aproximada de unos 3,280 habitantes en una extensión territorial de 42.6 manzanas. Finalmente, el asentamiento Arnoldo Alemán se encuentra ubicado en el sector sureste de la ciudad de Managua, Distrito VI, sobre la pista al municipio de Sabana Grande. La población de esta comunidad es aproximadamente de 8,527 habitantes distribuidos en una extensión territorial es de 724,003 m2 (103 manzanas).

\section{Breve caracterización socioeconómica general de las comunidades}

De acuerdo a la información recolectada y analizada por el MEM, se encontraron los siguientes resultados:

- Escasez de centros escolares y de asistencia médica.

- Falta de centros de recreación (áreas verdes, parques, canchas deportivas).

- Vías de acceso para transporte urbano en malas condiciones, predominando los caminos de tierra.

- Pocas fuentes de trabajo y salarios bajos.

- Predominio de bajo nivel académico.

- Baja cobertura efectiva de agua potable.

- Elevados índices de hacinamiento.

- Medios de comunicación y transporte accesibles (televisión, teléfonos, radio y buses).

- Porcentaje elevado de ingresos destinado a gastos en alimentos.

\section{Revisión de literatura}

La mayoría de los estudios relacionados con este tema de investigación utiliza diferentes enfoques metodológicos, variables, modelos y áreas de estudios, y por consiguiente, llegan a resultados heterogéneos. En nuestro caso, la importancia de revisarlos radica en que constituyen un importante cúmulo de referencias empíricas y teóricas. Por lo tanto, se procedió a revisar estudios sobre la materia, independientemente de la zona geográfica o el enfoque desarrollado. Éstos fueron insumos de gran valor al momento de extraer las variables dependientes de nuestra base de datos. 
Los estudios sobre los determinantes en la elección de tipo de combustible no son muy numerosos en Nicaragua, especialmente con un enfoque econométrico. Según Martínez Cuéllar (2003), los estudios sobre la demanda física de combustibles en los hogares no son abundantes. Las primeras investigaciones realizadas al respecto enfocan el análisis en la deforestación y degradación de los bosques e ignoran el problema energético.

La firma consultora "Multiconsult \& Cia. Ltda" realizó en el año 2006 la "Encuesta Nacional de Leña, 2005”, investigación que aborda la demanda física de leña. A partir de los datos encontrados en esa encuesta hemos podido fortalecer el análisis de nuestros resultados. Aunque su enfoque es descriptivo, nos brinda indicios importantes de los factores que pueden estar influyendo en la decisión de las familias.

\subsection{Demanda por tipo de combustible}

Para estimar los determinantes de la elección de GLP o leña, es preciso analizar la demanda de combustibles al interior de los hogares. Una primera aproximación consiste en evaluar variables que permiten medir la escasez del combustible. En la leña, distancia y tiempo de recolección, y el comportamiento de los hogares frente al número de hijos en la familia. Este enfoque no permite estimar la demanda física de GLP o de leña: tan sólo relaciona el tamaño del hogar con el consumo del combustible. La segunda aproximación determina los factores macroeconómicos que influyen sobre la demanda de combustible. Entre éstos se destaca la pobreza, el ingreso y su distribución. Una tercera aproximación determina factores microeconómicos, entre ellos: ingreso familiar, número de miembros del hogar, clima, factores culturales, género y edad.

Para la realización de esta investigación se utilizará la combinación de los tres enfoques por considerar necesario explorar el comportamiento de las familias seleccionadas.

\subsubsection{Demanda de combustible y el tamaño del hogar}

Dasgaputa (1993) atribuye la función de recolectar leña a la mujer -tarea que demanda gran parte de su tiempo- con apoyo de los niños. En su análisis, un hogar pequeño -pocos miembros- no sobrevive pues se requieren muchas manos para desempeñar las labores diarias, entre ellas la recolección de leña. En consecuencia, a mayor escasez de leña mayor será la demanda de niños y mayor el crecimiento poblacional.

Este estudio presenta el análisis de una zona rural, específicamente de los hogares que usan leña. En nuestro caso, los asentamientos se encuentran en la zona urbana y cerca de un 60\% usan GLP. No obstante, se considera de gran importancia incluir el tamaño del hogar para detectar si este factor ejerce influencia en la elección, aun cuando las condiciones del estudio sean diferentes.

Filmer y Pritchett (1996) encuentran que las familias destinan una parte significativa de su tiempo a recolectar leña y que son las mujeres y los niños los que realizan esta actividad. No obstante, no estiman la demanda física de leña, tan sólo utilizan proxies para medir su escasez. 


\subsubsection{Determinantes macroeconómicos}

La demanda de combustible está determinada por factores macroeconómicos tales como el nivel de pobreza e ingreso y su distribución, el avance tecnológico, el crecimiento poblacional y el grado de concentración de la población urbana.

La pobreza es uno de los determinantes más importantes de la demanda de leña. Los pobres del sector rural constituyen un grupo altamente dependiente de la leña para la preparación de alimentos, calentarse o generar iluminación. Los resultados de la EMNV 2005 (Instituto Nacional de Información de Desarrollo, 2005b) muestran que en Nicaragua el consumo de leña aumenta con el nivel de pobreza.

La leña es una excelente alternativa para la población pobre: en caso de no disponer de ingresos para adquirirla, simplemente buscan un lugar para recolectarla, por lo que su precio, cuando simplemente se recolecta, es cero ${ }^{2}$. Otro elemento muy importante ocurre con la tecnología empleada en la preparación de alimentos. Al cocinar con leña no se requiere de la compra de una estufa, tan sólo se necesita realizar una hoguera.

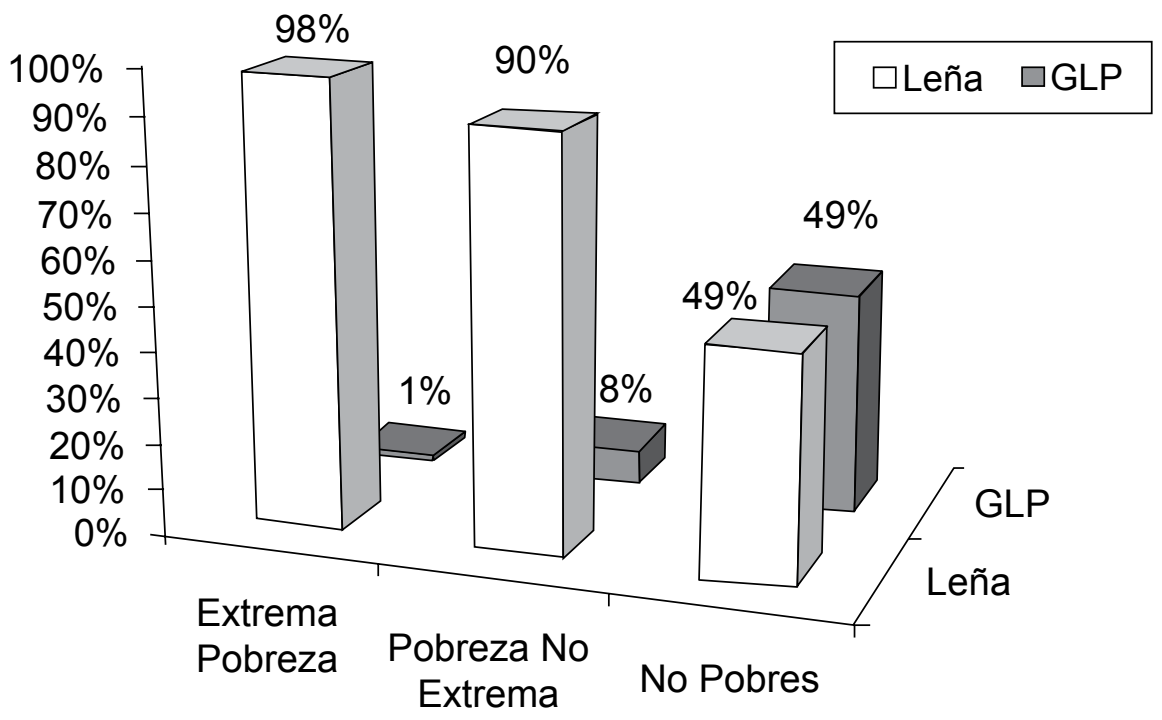

Ilustración 1. Tipo de combustible para cocinar y situación de pobreza Fuente: Autores en base a EMVN, 2005.

La demanda de combustible también depende del nivel de ingreso de los países: en la medida en que éstos se desarrollan, aumenta el consumo de combustibles comerciales y se reduce la dependencia de la leña. En Nicaragua, según Meza (2009) a pesar de registrarse una tasa de crecimiento sostenida en los volúmenes de venta de cilindros de GLP de $11.34 \mathrm{~kg}$ en los últimos 10 años, el consumo per cápita nacional se ha mantenido prácticamente constante, cercano a 16 lbs. de GLP al año. Es decir que el crecimiento en los volúmenes de ventas no implica que se esté cocinando un mayor número de alimentos por persona con GLP. 
El grado de urbanismo y el avance tecnológico también determinan la elección de tipo de combustible. El proceso de urbanización afecta los patrones de consumo de energía, en especial de la leña. El precio no es el único determinante que incide sobre el comportamiento del consumidor: los hogares urbanos prefieren combustibles más limpios y eficientes, aunque la leña sea la opción más barata. De igual manera, estos hogares acceden a fuentes de energía a las que no tenían acceso, como por ejemplo, la energía eléctrica. La disponibilidad de aparatos electrónicos, como computadores y otras aplicaciones eléctricas, cambia también las preferencias de los hogares, en particular de los sectores más ricos de la población.

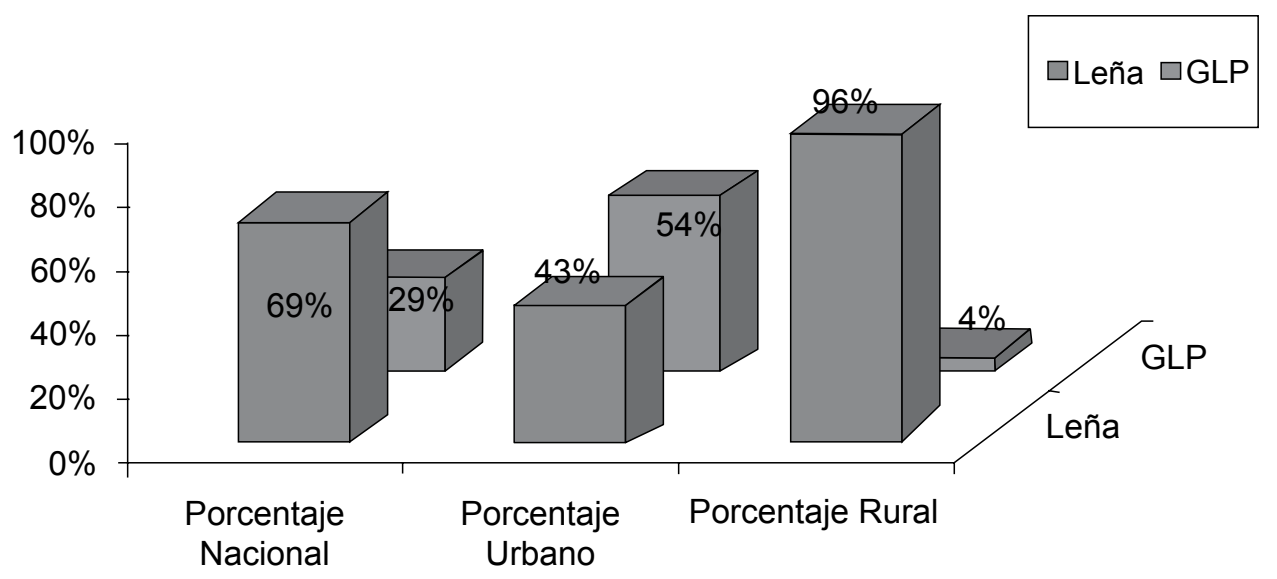

Ilustración 2. Elección del tipo de combustible a nivel nacional, urbano y rural en Nicaragua

Fuente: Autores en base a EMNV, 2005 (INIDE, 2005b).

\subsubsection{Determinantes microeconómicos}

Leach y Gowen (1987) analizan los factores que inciden en la demanda de combustible de los hogares e identifican su relación con el ingreso, tamaño del hogar, clima y precipitación (para calentar la vivienda), y con factores culturales tales como: dieta, hábitos al cocinar, iluminación, número de comidas diarias, rituales y costo de aparatos electrónicos. Los autores identifican que el factor que más incide sobre la demanda de combustible, inclusive por encima del ingreso, es el tamaño del hogar. Sostienen que a mayor tamaño del hogar, mayor ingreso total y mayor demanda de energía -por ser más los miembros.

Sobre la elección del tipo de combustible también incide el género, edad y educación de los miembros del hogar. Según la FAO (1997) las mujeres y niños son los principales recolectores de leña y la mujer es la que toma la decisión sobre qué tipo de combustible utilizar para preparar los alimentos, en especial en los países en desarrollo.

La mujer sacrifica tiempo en la recolección de la leña, tiempo que podría utilizar generando algún ingreso adicional que permita adquirir combustibles alternativos (Pacundan, 1997). En el caso de Nicaragua existe evidencia de que este trabajo no lo realizan las mujeres sino los hombres y los niños (Instituto Nacional de Información de Desarrollo, 2005b). 


\subsection{Estudios empíricos}

Existen dos tipos de estudios empíricos sobre la demanda de combustible: el descriptivo y el econométrico. Entre los trabajos descriptivos se destacan la Encuesta Nacional de Leña, 2005 (Multiconsult \& Cía. Ltda., 2005) en Nicaragua y los estudios de Briscoe (1979) y French (1981) a nivel internacional. Para la estimación econométrica sobresalen los de Pitt (1985), Kumar y Hotchkiss (1988), Stevenson (1989), Hamacher, Hyde y Joshee (1993), Bluffstone (1995), y Hamacher, Hyde y Kanel (1996), todos ellos realizados a nivel internacional.

Uno de los estudios más minuciosos es el de Briscoe (1979), quien evalúa el uso del recurso en una aldea en Bangladesh. Su principal conclusión es que la leña utilizada en los hogares no proviene del tronco principal de los árboles sino de pequeñas ramas y hojas, lo que implica una menor presión sobre el recurso forestal. Este resultado es similar a los resultados encontrados en la Encuesta Nacional de Leña en Nicaragua, 2005 (Multiconsult \& Cía. Ltda., 2005).

French (1981), por su parte, encuentra que las labores realizadas en los hogares donde se consume energía básicamente son: cocinar, calentar agua e iluminar. Adicionalmente, identifica el combustible utilizado y la intensidad de su uso en cada oficio. También indaga sobre la recolección de leña, la distancia a ser recorrida para tal efecto, si es comprada o recogida y el tiempo gastado en este oficio, el tipo de estufas utilizadas y el número de comidas diarias. Así, pudo calcular la eficiencia en el uso del combustible.

Kumar y Hotchkiss (1988) miden el tiempo destinado por las mujeres a la recolección de leña, labores agrícolas y de nutrición de la familia. Para tal efecto, evalúan la demanda de leña en términos del papel de la mujer y los hijos en la recolección de leña, la tecnología utilizada en las estufas y el ingreso agrícola.

Hamacher, Hyde y Joshee (1993) concluyen que la leña es un bien inferior en base a la estimación del consumo y la producción de la misma. Hamacher, Hyde y Kanel (1996) analizan la demanda de leña con base en la disponibilidad de tiempo de los miembros del hogar y de las alternativas de trabajo e ingresos: el que los miembros del hogar cuenten con la posibilidad de trabajar y reciban un salario, no necesariamente significa que demanden combustibles diferentes a la leña, así no la tengan que recoger ellos mismos, sino que se abastezcan en algún mercado.

\section{Marco teórico}

En la literatura existen dos enfoques para la interpretación estructural de los modelos de elección discreta. El primero hace referencia a la modelización de una variable latente a través de una función índice, que trata de modelizar una variable inobservable o latente. El segundo de los enfoques permite interpretar los modelos de elección discreta bajo la teoría de la utilidad aleatoria, de tal manera que la alternativa seleccionada en cada caso será aquella que maximice la utilidad esperada (Moral, 2003).

En nuestro caso se utilizará el segundo enfoque, ya que suponemos que la alternativa seleccionada del tipo de combustible, en este caso leña o gas, maximiza la utilidad esperada 
de las familias. Este segundo enfoque para la interpretación de los modelos de respuesta dicotómica es el que hace referencia a la modelización a través de la formulación de una utilidad aleatoria. Una familia debe adoptar una decisión que le permita elegir entre dos alternativas excluyentes, el GLP o la leña como combustible primario, lo que hará maximizando la utilidad esperada que le proporciona elegir una de las alternativas.

Es decir, el hogar elegirá una de las dos alternativas dependiendo de que la utilidad que le proporcione dicha decisión sea superior a la que le proporcione su complementaria. Un hogar racional decidirá consumir un combustible si la utilidad que deriva de esta alternativa supera la utilidad de escoger otra alternativa.

Un hogar escoge la leña o GLP basándose en consideraciones monetarias ${ }^{3}$, socio-económicas, familiares, geográficas y culturales.

La formulación del modelo bajo esta teoría parte del supuesto de que la utilidad derivada de una elección, Ui0 o Ui1, es función de las variables explicativas de dicha decisión, que son las características propias de cada una de las alternativas de elección y las características propias de la familia, de manera que suponiendo linealidad en las funciones, se tiene:

$$
\begin{aligned}
& U_{i 0}=\alpha_{0}+X_{i 0} \beta+\varepsilon_{i 0} \\
& U_{i 1}=\alpha_{1}+X_{i 1} \beta+\varepsilon_{i 1}
\end{aligned} \mid
$$

donde los eij recogen las desviaciones aleatorias que los agentes tienen respecto a lo que sería el comportamiento del agente medio. El agente i elegirá la opción 1 si la utilidad de esa decisión supera la de la opción 0 y viceversa, de manera que,

$$
Y_{i}=\left\{\begin{array}{ccc}
1 & \text { si } & U_{i 1}>U_{i 0} \\
0 & \text { si } & U_{i 1}<U_{i 0}
\end{array} \mid\right.
$$

y el modelo dicotómico quedaría definido por,

$$
\operatorname{Pr} o b\left(Y_{i}=1\right)=\operatorname{Pr} o b\left(U_{i 1}>U_{i 0}\right)=\operatorname{Pr} o b\left(U_{i 1}-U_{i 0}>0\right)=F\left(X_{i} \beta\right)
$$

Según la función asociada a la perturbación aleatoria eij (que será la función de distribución, $\mathrm{F}(\mathrm{Xi} \beta)$ que se suponga siga dicha probabilidad), sea una función de distribución uniforme, la función de distribución de la normal tipificada o la de la curva logística, se obtienen el Modelo Lineal de Probabilidad Truncado, el Probit o el Logit, respectivamente.

Dado que en el modelo lineal de probabilidad existe la posibilidad de que la probabilidad esté por fuera del rango $(0,1)$, se utilizó un modelo logístico binario. 


\section{Metodología}

\section{a. El modelo logístico binario}

El modelo de elección de tipo de combustible para la cocción de alimentos está formulado de forma que mida la subjetividad de las familias respecto a su decisión/elección de tipo de combustible. Para esto se ha utilizado el modelo econométrico de regresión logística binaria con variable dependiente "Y" con probabilidad "n" donde para el caso ith

$\mathrm{p}_{i}=\frac{\exp \left(\mathrm{h}_{i}\right)}{1+\exp \left(\mathrm{h}_{i}\right)}$

ó

$\ln \left(\frac{\mathrm{p}_{i}}{1-\mathrm{p}_{i}}\right)=\mathrm{h}_{i}=X_{i}^{\prime} \mathrm{b}$

Por lo tanto, la función de probabilidad de ocurrencia "l" para "n" observaciones y, .., yn, con probabilidades $\pi 1, \ldots, \pi \mathrm{n}$ y casos ponderados $\mathrm{w} 1, \ldots, \mathrm{wn}$, se puede escribir de la siguiente forma:

$$
l=\prod_{i=1}^{n} \mathrm{p}_{i}^{w_{i} y_{i}}\left(1-\mathrm{p}_{i}\right)^{w_{i}\left(1-y_{i}\right)}
$$

Sigue que el logaritmo de "l" es

$$
L=\ln (l)=\sum_{i=1}^{n}\left(w_{i} y_{i} \ln \left(\mathrm{p}_{i}\right)+w_{i}\left(1-y_{i}\right) \ln \left(1-\mathrm{p}_{i}\right)\right)
$$

Y la derivada parcial con respecto a los parámetros $\beta$ j es

$$
L_{X_{j}}^{*}=\frac{\partial L}{\partial \mathrm{b}_{j}}=\sum_{i=1}^{n}\left(w_{i}\left(y_{i}-\mathrm{p}_{i}\right) x_{i j}\right)
$$

Donde:

$\mathrm{n}=\quad$ Número de casos observados

$\mathrm{x}=\quad$ Matriz $\mathrm{n} \mathrm{x} \mathrm{p}$ con elementos xij, el valor observado del caso iésimo caso del parámetro jésimo.

$\beta=\quad$ vector $\mathrm{p} \times 1$ con elementos $\beta \mathrm{j}$, el coeficiente para el jésimo parámetro

$1=$ Función de probabilidad 
$\mathrm{L} \quad=\quad$ Logaritmo de la función de probabilidad

$\pi \mathrm{i}=\quad$ Probabilidad del iésimo caso que experimenta el evento de interés

$\eta \dot{i}=$ Ocurrencia del evento para el iésimo caso

\section{b. El modelo ajustado}

La ecuación para la elección del tipo de combustible es la ecuación 10 y 11.

El modelo asume que la variable dependiente "Z" está relacionada linealmente con las variables independientes expresada de la siguiente manera.

$$
Z_{i}=b_{0}+b_{1} x_{i 1}+b_{2} x_{i 2} \ldots+b_{p} x_{i p}
$$

Sin embargo, como la variable " $Z$ " no se puede observar pues representa la linealización de la función logística, la regresión debe ajustarse relacionando los predictores (variables independientes) a la probabilidad de interés sustituyendo " $Z$ " por la siguiente ecuación.

$$
\mathrm{p}_{i}=\frac{1}{1+e^{-\left(b_{0}+b_{1} x_{i 1}+b_{2} x_{i 2} \ldots+b_{p} x_{i p}\right)}}
$$

donde :

xij = es el jésimo predictor o variable independiente para el iésimo caso.

bj = es el jésimo coeficiente

$\mathrm{p}=$ es el número de predictores o variables independientes.

Así los coeficientes de regresión son estimados a través del método iterativo de máxima verosimilitud.

\section{c. Supuestos}

El desarrollo del modelo de elección del tipo de combustible parte de los siguientes supuestos:

1. Las variables independientes son normalmente distribuidas.

2. La elección de las familias está contenida en las variables explicativas.

3. La inversión en estufas en base a GLP, el cilindro, el hidrocarburo y demás artefactos está en función del ingreso.

A la luz de los supuestos antes expuestos, los resultados aquí presentados son una aproximación del posible comportamiento de las familias en los barrios estudiados, cuyos pobladores responden a sus expectativas sobre su satisfacción. Este comportamiento se mide en términos probabilísticos para ilustrar la elección del tipo de combustible. 


\section{Datos}

A partir de la revisión de literatura y busca de las variables que permiten explicar la elección del tipo de combustible para la cocción de alimentos, se procedió a seleccionar de la encuesta un grupo de variables que en primera instancia se presumía pudiesen explicar la decisión de elegir leña o GLP como combustible primario.

Cuadro 1. Descripción de las variables

\begin{tabular}{|c|c|c|c|c|c|c|}
\hline Variables & Descripción & & Frecuencia & Media & Mínimo & Máximo \\
\hline \multirow{2}{*}{$\begin{array}{l}\text { Combustible } \\
\text { (DEPENDIENTE) }\end{array}$} & \multirow{2}{*}{$\begin{array}{l}\text { Tipo de combustible primario } \\
\text { elegido en el hogar }\end{array}$} & 0-Leña & 145 & --- & ---- & ----- \\
\hline & & 1-GLP & 258 & ----- & ----- & ----- \\
\hline \multirow{2}{*}{ Trabajajef } & \multirow{2}{*}{ Trabaja el jefe de hogar } & 1-Si & 301 & ----- & ------ & ---- \\
\hline & & 0 -No & 103 & ----- & ---- & ---- \\
\hline Nios & \multicolumn{2}{|l|}{ Número de niños menores de 10 años } & 400 & 2 & 0 & 11 \\
\hline Edadjef & \multicolumn{2}{|l|}{ Edad del jefe del hogar } & 394 & 40 & 18 & 81 \\
\hline Notrabajando & \multicolumn{2}{|c|}{$\begin{array}{l}\text { Número de miembros del hogar que declararon } \\
\text { tener empleo remunerado }\end{array}$} & ---- & 2 & 0 & 7 \\
\hline Ingrereal & \multicolumn{2}{|c|}{ Ingreso Real Familiar Mensual (C\$ 1999) } & 400 & $2,275.389$ & 360.52 & $14,240.65$ \\
\hline \multirow{2}{*}{ Sexojef } & \multirow{2}{*}{ Género } & 1-Masculino & 201 & ---- & ----- & ---- \\
\hline & & 0-Femenino & 196 & ---- & ----- & ----- \\
\hline \multirow{2}{*}{ Localizacion } & \multirow{2}{*}{$\begin{array}{l}\text { Departamento al que pertenece el } \\
\text { barrio }\end{array}$} & 1-Managua & 221 & -..-- & ---- & ---- \\
\hline & & 0-León & 183 & -ב- & ב-- & ב-- \\
\hline \multirow{2}{*}{ Penergae } & \multirow{2}{*}{$\begin{array}{l}\text { Paga la vivienda el servicio de } \\
\text { energía eléctrica }\end{array}$} & 1-Si & 223 & - & - & ----- \\
\hline & & 0-No & 180 & - & - & ----- \\
\hline Nopers & \multicolumn{2}{|l|}{ Número de personas en el hogar } & 404 & 5 & 1 & 24 \\
\hline \multirow[t]{2}{*}{ Estadoconyugal } & \multirow{2}{*}{$\begin{array}{l}\text { Estado Conyugal del Jefe del } \\
\text { Hogar }\end{array}$} & $\begin{array}{l}\text { 0- Soltería, } \\
\text { v i u d e z , } \\
\text { separación }\end{array}$ & 72 & ---- & ---- & ---- \\
\hline & & $\begin{array}{l}1 \text { - U n i ó n } \\
\text { Matrimonio }\end{array}$ & 320 & 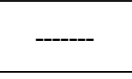 & - & --- \\
\hline \multirow{5}{*}{ Educacionjef } & \multirow{5}{*}{ Educación del Jefe del hogar } & 0- Ninguno & 34 & --- & ---- & ----- \\
\hline & & 1- Primaria & 141 & ---- & ----- & ---- \\
\hline & & 2- Secundaria & 165 & -ב-- & ----- & - --- \\
\hline & & 3- Técnico & 18 & --- & ----- & --- \\
\hline & & 4- Universitario & 42 & --- & ----- & ---- \\
\hline
\end{tabular}

Fuente: Autores

La variable dependiente llamada "combustible", dicótoma, toma forma de 0 cuando se utiliza leña como combustible para cocción de los alimentos y 1 cuando se utiliza GLP.

Se seleccionaron 11 variables explicativas entre las cuales figuran:

- Ingreso familiar real $(\mathrm{C} \$=1999)$

- Paga o no la vivienda el servicio de energía eléctrica como variable proxi al precio de un bien sustituto, en este caso la cocina eléctrica 
- Localización del asentamiento para determinar el efecto geográfico en la demanda de GLP o leña

- Edad del jefe del hogar como variable proxi a los hábitos de cocción de los alimentos

- Educación del jefe del hogar

- Conjunto de variables socio-económicas

\section{Discusión de resultados}

\subsection{Primera estimación del modelo logístico binario}

Cuadro 2. Parámetros estimados de primera regresión del modelo ${ }^{4}$

\begin{tabular}{|c|c|c|c|c|c|c|}
\hline Variables & $\begin{array}{l}\text { Tipo de } \\
\text { variable }\end{array}$ & Signo esperado & \begin{tabular}{|l|} 
Coeficiente estimado \\
Logit
\end{tabular} & $\mathrm{P}>|\mathrm{z}|$ & Significancia & Conclusión \\
\hline Combustible & Dicótoma & ------ & ------- & ----- & ------ & Variable dependiente \\
\hline Constante & ----- & & 0.1485 & 0.837 & & $\begin{array}{l}\text { Constante en el } \\
\text { modelo incluir }\end{array}$ \\
\hline Trabajajef & Dicótoma & Positivo & -0.1111 & 0.709 & & Excluir \\
\hline Nios & Continua & Negativo & -0.0368 & 0.784 & & Excluir \\
\hline Edadjef & Continua & Negativo & -0.0204 & 0.077 & 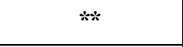 & Incluir \\
\hline Notrabajando & Continua & ------- & -0.2512 & 0.182 & $*$ & Incluir \\
\hline Ingrereal & Continua & Positivo & 0.0003 & 0.001 & $* *$ & Incluir \\
\hline Sexojef & Dicótoma & Negativo & -0.1445 & 0.590 & & Excluir \\
\hline Localizacion & Dicótoma & - & 0.4235 & 0.092 & $*$ & Incluir \\
\hline Penergae & Dicótoma & Positivo & 0.2754 & 0.260 & & Excluir \\
\hline Nopers & Continua & ----- & -0.0748 & 0.409 & & Excluir \\
\hline Estadoconyuga & Dicótoma & ------- & -0.1579 & 0.650 & & Excluir \\
\hline Educacionjef & Categórica & Positivo & 0.6816 & 0.000 & $* *$ & Incluir \\
\hline \multicolumn{3}{|c|}{ Log likelihood $=-211.63$} & \multicolumn{3}{|l|}{ Prob $>$ chi $2=0.0000$} & $\begin{array}{l}* \text { Indica significativo } \\
\text { al } 90 \% \text { de confianza }\end{array}$ \\
\hline \multicolumn{3}{|c|}{ LR $\operatorname{chi} 2(11)=62.70$} & \multirow{2}{*}{\multicolumn{3}{|c|}{ Número de observaciones $=373^{5}$}} & $\begin{array}{l}\text { Indica significativo } \\
\text { al } 95 \% \text { de confianza }\end{array}$ \\
\hline \multicolumn{3}{|c|}{ Pseudo R2 = 0.1290} & & & & $\begin{array}{l}\text { al Indica significativo } \\
\text { a9\% de confianza }\end{array}$ \\
\hline
\end{tabular}

Fuente: Autores

Esta primera estimación del modelo nos muestra resultados sumamente interesantes. La variable "Trabajef", (-0.1111) que indica la condición de empleo o desempleo del jefe del hogar, no es significativa al momento de elegir el tipo de combustible, siendo factores como el volumen de ingresos o la localización más influyentes.

A pesar de que los niños son de los miembros de la familia que más sufren el impacto de la ingesta del humo y que generalmente generan un flujo de gastos considerable en la economía familiar, la variable número de niños menores a 10 años "Nios", con un coeficiente de -0.0368 , tiene el signo esperado pero no es importante al momento de la elección del combustible. Esto podría explicarse por un desconocimiento de parte de los padres de los efectos negativos de la ingesta de humo en sus hijos, posibilitado, naturalmente, por los 
bajos niveles de escolaridad de los jefes del hogar. En este sentido, la mayoría de los jefes de familia no tienen un nivel de escolaridad superior a la primaria.

La edad del jefe del hogar "edadjef", con un coeficiente de -0.0204, tuvo el signo esperado y es significativa al 90 por ciento. Los hogares con jefes de hogar en estado de edad avanzado tienen mayor probabilidad de usar leña para cocinar que aquellos jefes jóvenes. Este resultado viene a ratificar la importancia del factor cultural en los hábitos de cocción de alimentos.

Al igual que en los resultados encontrados por Hamacher, Hyde y Kanel (1996), la variable número de miembros trabajando "notrabajando" (-0.2512) tiene signo negativo y no es significativa. Es decir, el que los miembros del hogar cuenten con la posibilidad de trabajar y recibir ingresos no necesariamente significa que demanden un combustible diferente de la leña. Una posible explicación es la baja propensión marginal al ahorro, que impide acumular suficientes recursos para invertir en estufas y utensilios.

El coeficiente del ingreso familiar mensual real "ingrereal" (0.0003) tiene signo positivo y es significativo al 99 por ciento. Es decir, incrementos en el ingreso familiar real elevan las probabilidades de sustituir la leña por el GLP como combustible.

Se incluyó la variable género en el modelo con el nombre de "sexojef" con el objetivo de determinar si hombres jefes de hogar y mujeres jefas de hogar tienden a elegir con criterios diferentes. Los roles y prácticas culturales vinculadas al género tienden a responsabilizar a mujeres y niñas de la mayoría de los oficios domésticos. Se estima que en los países en desarrollo ocurren 1,6 millones de muertes al año -de las cuales el 60 por ciento son femeninas- asociadas con la inhalación de humo en sitios cerrados por el uso de combustibles sólidos (OMS, 2002).

A pesar de esta situación, el coeficiente de la variable de género (-0.1445) tiene el signo negativo esperado, indicando que las mujeres jefas de hogar tienden a utilizar con menor frecuencia la leña, pero éste no es significativo. Este resultado apunta a que a pesar de que las mujeres sufren en mayor medida las consecuencias de utilizar leña, el hecho que el hogar tenga un cabeza de familia femenino, no necesariamente influye en la elección de GLP. El desconocimiento de los efectos negativos de la ingesta de humo y factores culturales y económicos impiden que muchas mujeres puedan sustituir la leña por el GLP.

El coeficiente de la "localización" (0.4235) es de signo positivo y registra una significancia al 90 por ciento. Estos resultados muestran, ceteris paribus, que los asentamientos ubicados en Managua tienen mayor probabilidad de utilizar GLP que los de León.

El coeficiente de la variable "penergae" (0.2754) correspondiente a si la vivienda paga o no paga su servicio de energía eléctrica, tiene signo positivo y no es estadísticamente significativo. La lógica económica indicaría que al no haber un "regulador de consumo de energía eléctrica", como el precio de la misma (debido a que no pagan el servicio), los habitantes podrían sustituir la leña por cocinas eléctricas ya que el costo de operación de las mismas sería prácticamente cero. 
No es necesario estimar este modelo para encontrar que los habitantes declararon no utilizar este tipo de cocinas ${ }^{7}$. Sin embargo, resulta sospechoso este resultado y debe ser interpretado con cautela ${ }^{8}$.

En el caso del número de personas en el hogar, "nopers", el coeficiente (-0.0748) no es significativo, mas el signo es el indicado. A mayor número de personas en el hogar, dados los bajos ingresos, la presión ejercida por los gastos en alimentación, vestimenta, transporte y servicios básicos provoca en muchas familias una situación de iliquidez.

El estado conyugal del jefe del hogar, "estadoconyugal", no resultó estadísticamente significativo. No obstante, se decidió incluir la variable debido a que la irresponsabilidad paterna ha demostrado, en algunos casos, ser un catalizador en el empeoramiento de las condiciones económicas, nutricionales y sociales de las familias (ver Vargas, 2001).

Tal como se esperaba, la educación del jefe del hogar, "educacionjef”, es determinante en la elección del tipo de combustible. El coeficiente 0.6816 indica que a mayor nivel de escolaridad la probabilidad de seleccionar GLP se incrementa significativamente.

\subsection{Resultados de segunda estimación del modelo}

Luego de correr un primer modelo se procedió a ajustar en base a criterios de significancia estadística, de lógica socio-económica y de bondad de ajuste (ver anexo 2). Se seleccionaron las variables con un significancia igual o mayor al 90 por ciento y que presentase el signo esperado. A partir de esta selección se inicio un proceso iterativo que permitiera capturar los factores determinantes en la elección.

Los resultados del modelo de elección del tipo de combustible se muestran en el Cuadro 3.

Cuadro 3. Parámetros estimados del modelo de elección del tipo de combustible para cocción de alimentos

\begin{tabular}{|c|c|c|c|c|c|c|}
\hline Variables & $\begin{array}{l}\text { Tipo de } \\
\text { variable }\end{array}$ & $\begin{array}{l}\text { Signo } \\
\text { esperado }\end{array}$ & $\begin{array}{c}\text { Coeficiente } \\
\text { estimado Logit }\end{array}$ & $\mathrm{P}>|\mathrm{z}|$ & Significancia & Conclusión \\
\hline Combustible & Dicótoma & - & 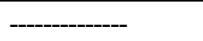 & 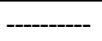 & 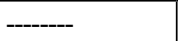 & Variable dependiente \\
\hline Constante & ------ & & -0.2416517 & 0.685 & -- & Constante en el modelo incluir \\
\hline Edadjef & Continua & Negativo & -0.0170022 & 0.097 & $*$ & Incluir \\
\hline Ingrereal & Continua & Positivo & 0.0003573 & 0.000 & 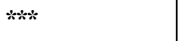 & Incluir \\
\hline Localizacion & Dicótoma & 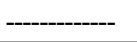 & 0.4557921 & 0.059 & $*$ & Incluir \\
\hline Nopers & Continua & Negativo & -0.1318962 & 0.025 & * & Incluir \\
\hline Educacionjef & Categórica & Positivo & 0.6952672 & 0.000 & $w *$ & Incluir \\
\hline \multicolumn{3}{|c|}{ Log likelihood $=-220.71158$} & \multicolumn{3}{|c|}{ Prob $>$ chi $2=0.0000$} & $\begin{array}{l}\text { * Indica significativo al 90\% de } \\
\text { confianza. }\end{array}$ \\
\hline \multicolumn{3}{|c|}{ LR $\operatorname{chi} 2(5)=63.66$} & \multirow{2}{*}{\multicolumn{3}{|c|}{ Número de observaciones $=388^{9}$}} & $\begin{array}{l}\text { Indica significativo al } 95 \% \text { de } \\
\text { confianza. }\end{array}$ \\
\hline \multicolumn{3}{|c|}{ Pseudo R2 $=0.1260$} & & & & $\begin{array}{l}\text { Indica significativo al 99\% de } \\
\text { confianza. }\end{array}$ \\
\hline
\end{tabular}

Fuente: Autores 
El modelo muestra todos los coeficientes de las variables seleccionadas con los signos esperados y estadísticamente significativos al menos al 90 por ciento. Un aspecto notable es la significancia estadística que adquirió el coeficiente de la variable número de personas en el hogar "nopers" (-0.1318962), brindando una base más sólida para analizar los comportamientos de las familias desde un enfoque presupuestario y energético.

\subsection{Estimación de los efectos marginales y elasticidades en el modelo logístico ajustado}

Luego de la selección del modelo se procedió a calcular los efectos marginales y las elasticidades de los coeficientes, de manera que el análisis pueda efectuarse con mayor claridad, concisión y utilidad. Los resultados se ordenaron en el siguiente cuadro:

Cuadro 4. Resultados de los efectos marginales y elasticidades

\begin{tabular}{|c|c|c|c|c|c|c|}
\hline Variables & \begin{tabular}{ll|} 
Tipo de \\
variable
\end{tabular} & Signo esperado & Efecto marginal & $\mathrm{P}>|\mathrm{z}|$ & Elasticidad & $\mathrm{P}>|\mathrm{z}|$ \\
\hline Combustible & Dicótoma & 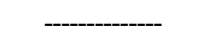 & 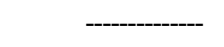 & - & --_---' & \\
\hline Constante & - & 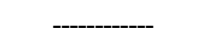 & - & - & - & \\
\hline Edadjef & Continua & Negativo & -0.0037192 & 0.097 & -0.2164751 & 0.099 \\
\hline Ingrereal & Continua & Positivo & 0.0000782 & 0.000 & 0.2645412 & 0.000 \\
\hline Localizacion & Dicótoma & - & No tiene & ----- & 0.0797354 & 0.059 \\
\hline Nopers & Continua & Negativo & -0.0288521 & 0.024 & -0.2029385 & 0.025 \\
\hline Educacionjef & Categórica & Positivo & No tiene & ---- & 0.3921079 & 0.000 \\
\hline
\end{tabular}

Una diferencia de edad de un año entre un jefe de hogar mayor frente a otro, un año menor, representa una disminución de 0.003 de probabilidades de que en esa familia se elija GLP. La edad del jefe del hogar captura en parte el elemento cultural en los hábitos de cocción de alimentos. Muchos de los jefes de familia de edad avanzada declaran preferencia por el sabor de los alimentos cocinados en leña. Adicionalmente, argumentan que la velocidad del fogón de leña para cocinar los alimentos es mayor que la de la cocina, posibilitando la realización otras actividades durante el día.

Por otro lado, algunos jefes de hogar son pensionados. El monto mensual de las pensiones es relativamente bajo en Nicaragua. Por tanto, los ingresos de familias que dependen de un cabeza de familia pensionado, tienden a ser menores a los de un jefe en edad de trabajar y empleado, imponiendo una restricción de ingresos a la canasta de bienes de consumo familiar.

En este sentido, incrementos en 1\% en el ingreso familiar real mensual mejoran en cerca de 26\% las probabilidades de sustituir la leña por el GLP. El ingreso promedio mensual en términos nominales de las familias en los asentamientos es de 5049.08 córdobas. La inversión en la adquisición de una cocina sencilla de cuatro quemadores, el envase del GLP (cilindro), la manguera de transporte del cilindro a la conexión de la cocina y el hidrocarburo (líquido) ${ }^{10}$ es cercana a los 1,660 córdobas ${ }^{11}$. 
Esta cifra implica que una familia promedio necesita destinar cerca del $33 \%$ de su ingreso familiar mensual a la transformación de su matriz energética de cocción de alimentos. Esto implica una elevada inversión inicial para muchas de estas familias que destinan parte importante de sus ingresos a financiar alimentos y servicios básicos. ${ }^{12}$

La ubicación departamental de los asentamientos es determinante en la elección del combustible. Las familias de los asentamientos de León son dos veces más propensas a utilizar leña que las familias establecidas en Managua, manteniendo el resto de factores inalterados. Esto se explica mediante dos factores:

a) Debilidad de las redes de comercialización de GLP en el departamento de León.

La ciudad de Managua, como ciudad capital, concentra la mayor cantidad de centros de distribución de GLP envasado en el país. Las redes de comercialización detallistas (pulperías) y distribuidores minoristas ("Centros Express") son extensas en la mayoría de barrios y asentamientos de la capital. En cambio, la ciudad de León cuenta con una menor oferta de puestos de venta de GLP, en especial en la modalidad de centros detallistas (pequeñas pulperías ubicadas en los barrios), que son las más frecuentadas por los consumidores para abastecerse. Por esta razón, el acceso a GLP presenta mayores facilidades en los asentamientos de la ciudad de Managua.

b) Los precios de la leña son más elevados en el departamento de Managua.

Según la Encuesta Nacional de Leña (Multiconsult \& Cía. Ltda., 2005), los precios de la leña en la ciudad de Managua son los más altos en Centroamérica. Si bien, no se aborda el aspecto del precio en este estudio por motivos de ausencia de datos, la situación de precios más bajos en la ciudad de León en comparación con la ciudad de Managua, permite a las familias de los asentamientos de León acceder a una leña más barata.

El coeficiente de la variable "nopers" o número de miembros de la familia, indica que la adición de un nuevo miembro a la familia representa una disminución del tres por ciento en las probabilidades de sustituir GLP por la leña. Un nuevo miembro en la familia representa un flujo de gastos que generalmente no es compensado con incrementos en los ingresos ${ }^{13}$.

Esta situación produce tensión en el presupuesto familiar e impide ahorrar, provocando que las familias elijan un combustible monetariamente más "barato" como es la leña. Otro elemento importante es que el tiempo de duración de un cilindro de GLP de $11.34 \mathrm{~kg}$ en un hogar con muchos miembros es menor. Un número mayor de personas requiere cocinar un mayor volumen de alimentos y la utilización de una mayor cantidad de quemadores o "llamas". Esto implica la necesidad de comprar el hidrocarburo con mayor frecuencia, induciendo a las familias a utilizar la leña como combustible principal, ya que pueden cocinar sus alimentos gastando diario en la leña montos mucho menores a lo que representaría la adquisición de un cilindro de GLP. Los datos de la encuesta indican que el gasto diario en leña ronda entre los 5 y los 10 córdobas diarios.

Finalmente, el nivel de escolaridad del jefe de familia demuestra ser un factor determinante en la elección de combustibles. Un hogar que cuenta con un jefe de familia con un grado 
de escolaridad universitario, es ocho veces menos propenso a utilizar leña para cocinar que una persona analfabeta. La educación permite elevar la productividad del trabajo, lo que incide en los salarios e ingresos percibidos. Así mismo, se adquiere conciencia de los riesgos para la salud que significa la ingesta de humo para los miembros de la familia y la degradación ambiental al que contribuye el uso de leña para cocinar.

\section{Conclusiones}

Los resultados de este estudio muestran que factores considerados importantes en estudios similares no son significativos en las decisiones de las familias de estos barrios de Managua y León.

Las mujeres, niños y niñas son los más afectados por la ingesta de humo. Sin embargo, el género del jefe del hogar y la presencia de niños y niñas en la familia no son importantes. De la misma manera, la condición de empleo o desempleo del jefe del hogar y el número de personas trabajando no son variables significativas.

La cocina eléctrica es un sustituto del GLP y de la leña pero su utilización representa elevados costos en pago del servicio de energía eléctrica. Sin embargo, muchos de estos hogares no pagan el servicio de energía eléctrica (45\%) y a pesar de esta razón no se encontraron indicios de un efecto sustitución. Finalmente, la cantidad de personas trabajando en la familia no es importante al decidir el tipo de combustible a utilizar, aún cuando se esperarían flujos de ingresos familiares mayores y menor tiempo para adquirir la leña y encender el fuego.

Los factores determinantes en la elección son los siguientes:

Ingreso familiar real: Incrementos del uno por ciento elevan en 26 por ciento las probabilidades de que una familia elija GLP en vez de leña como combustible principal. Los aumentos en los ingresos favorecen la adquisición de estufas a base de GLP, el cilindro y demás utensilios. Además, permite resistir a las familias los incrementos en los precios del hidrocarburo.

Número de personas en el hogar: Un mayor número de personas en el hogar disminuye las probabilidades de uso de GLP. Esto se explica por el incremento en los gastos que una familia numerosa incurre en alimentación, transporte, vestimenta y demás necesidades. Esto impide ahorrar para adquirir las estufas de GLP, los cilindros vacíos y demás utensilios. En segundo lugar, esta posible situación de iliquidez les dificulta afrontar incrementos en los precios del hidrocarburo en caso de que ya posean la cocina, lo que se agrava debido a que el tiempo de duración de un cilindro de GLP disminuye en la medida en que se cocina un volumen mayor de alimentos y se usa un mayor número de quemadores.

Localización del asentamiento: Los asentamientos de León son dos veces más propensos a utilizar leña. Esto se debe a los siguientes elementos: poca oferta de GLP (menor número de centros de distribución detallista en los asentamientos Lino Argüello y Mariana Sansón de León), un precio de GLP envasado en cilindros mayor 
al de la ciudad de Managua (debido a los costos de transporte) y un precio de la leña menor al de Managua.

Educación del jefe del hogar: Mayores niveles educativos del jefe del hogar disminuyen las probabilidades de uso de leña. Esto podría explicarse por un mayor conocimiento de los efectos nocivos de la ingesta del humo sobre la salud de los miembros de la familia, así como los perjuicios medioambientales que ocasiona la tala de árboles para leña. Por otro lado, mayores niveles de educación favorecen los ingresos familiares y disminuyen las probabilidades de caer en el desempleo.

Edad del jefe del hogar: Los hogares con jefes de familia en edad avanzada tienen mayor probabilidad de usar leña, manteniendo inalterado el resto de factores. Este resultado sugiere cierta renuencia a utilizar GLP posiblemente alimentada por hábitos y concepciones culturales relacionadas con el sabor y la velocidad de cocción de los alimentos cocinados en leña y la supuesta "haraganería" que representa usar GLP.

Después de los resultados presentados en este artículo es posible afirmar que acciones como la del gobierno de Nicaragua, de transferir estufas con GLP envasado en cilindros, responden a una lógica acertada. Programas gubernamentales de este tipo elevan la posibilidad de que familias con ingresos bajos puedan sustituir la leña por un combustible de mayor eficiencia y menos contaminante como el GLP. El solventar la inversión inicial coadyuva a mejorar el bienestar de las familias, mitigar la degradación del suelo y disminuir la demanda de servicios de salud.

\section{Agradecimientos}

A la Dirección General de Mercado Eléctrico del Ministerio de Energía y Minas (MEM) por facilitarnos la base de datos.

\section{Notas}

1 Ver anexo 1.

2 Costo monetario sin incluir el costo de oportunidad.

3 Teóricamente la demanda se especifica en función del precio. Sin embargo, en este modelo no se utiliza el precio pues la encuesta no cuenta con la información precisa sobre el mismo. La encuesta provee datos sobre el valor gastado en la compra de ambos combustibles. Sin embargo, al tratar de obtener un precio, surgen problemas con la unidad de medida de compra de la leña, pues ésta varía de un lugar a otro en volumen y cantidad.

4 Se utilizó el programa econométrico Intercooled Stata-8.

5 El software depura las observaciones incompletas. Aun así, las 373 observaciones representan un nivel de confiabilidad superior al $95 \%$. Ver anexo 1.

6 Córdobas $=1999$

7 La selección de un modelo logístico binario y no un multinomial logit se debe a que los pobladores no declararon utilizar ningún otro combustible primario que no sea leña o GLP.

8 La encuesta con la que se realizó este estudio fue elaborada por el MEM con el objetivo de evaluar la capacidad de pago de los habitantes por el servicio de energía eléctrica, por tanto, puede sospecharse dado los elevados volúmenes de energía que consumen las cocinas eléctricas, que los encuestados pudiesen no declarar sus cocinas eléctricas. 
9 El software depura las observaciones incompletas. Aun así, las 388 observaciones representan un nivel de confiabilidad superior al $95 \%$. Ver anexo 1.

10 Según el Instituto Nicaragüense de Energía (2010) el GLP envasado en cilindros de $11.34 \mathrm{~kg}$ se cotiza en la segunda semana de enero de 2010 en unos 250 córdobas.

11 Estimación propia en base a precios de mercado. Estos precios se recopilaron a través de llamadas telefónicas a puestos de venta en Managua y León.

12 Esta situación se agrava debido a la dificultad para optar por créditos en tiendas de electrodomésticos.

13 En el período entre enero de 1994 y octubre de 2009 el salario real promedio nacional creció a tasas menores al uno por ciento.

14 A través de una serie de reformas del sector energético y de programas de subsidios, desde 1974 el Gobierno de Senegal ha contribuido a incrementar el consumo de GLP en 11\% anual. Esto se ha traducido en una reducción notable de la deforestación. En este sentido, en el 2002 el consumo de 100.000 toneladas de GLP evitó la deforestación de 40.500 hectáreas o la producción de 337.500 toneladas de carbón vegetal. Fuente: ENDA tercer mundo-Medio Ambiente y Desarrollo del Tercer Mundo

\section{Anexo 1. Fórmula para determinar la confiabilidad de la muestra}

$$
n=\frac{Z^{2} * p^{*} q^{*} N}{\left(N^{*} e^{2)}+\left(Z^{2} * p^{*} q\right)\right.}
$$

$\mathrm{Z}=1.96$, valor en la tabla de distribución Normal para el $95 \%$ de confianza;

$\mathrm{N}=$ es la población total $=2,500$ viviendas

p y q $=$ probabilidades complementarias de 0.5 ,

$\mathrm{e}=$ error de estimación $=0.05$

$\mathrm{n}=$ tamaño de la muestra $=360$

\section{Anexo 2. Función de Distribución Acumulativa FDLA}

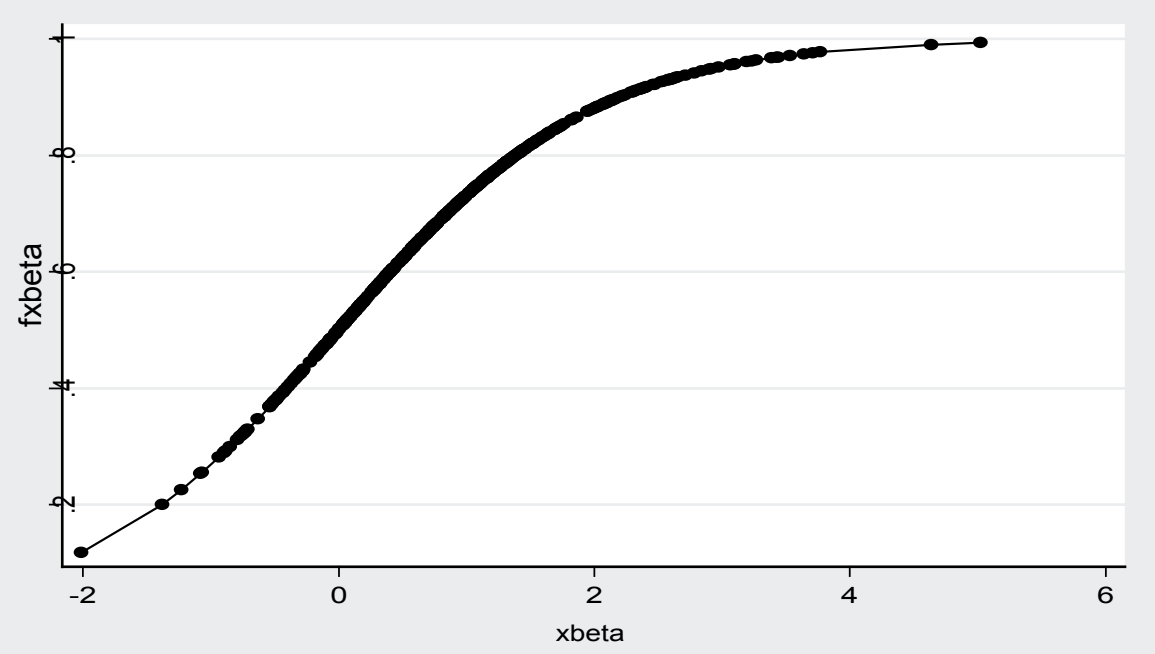

Fuente: Elaboración de los autores. 
Nótese que los efectos marginales debido a un cambio en xbeta no son constantes. El efecto parcial es más grande cuando xbeta es cero y más pequeño a medida que xbeta se acerca $\mathrm{a}-\infty \mathrm{y}+\infty$.

\section{Anexo 3. Bondad de ajuste del modelo}

$\checkmark$ Detalle de la proporción de observaciones predichas correctamente

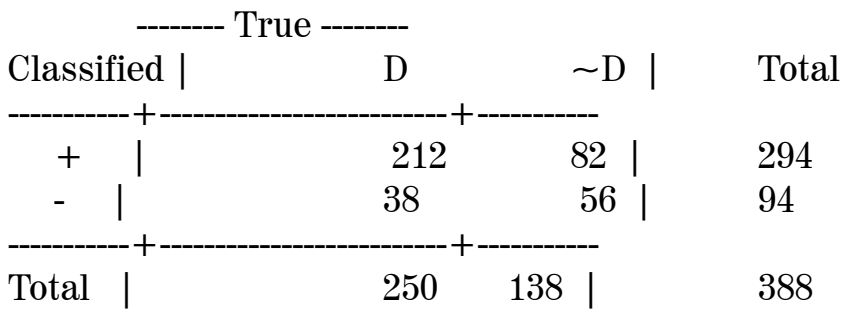

24 Classified + if predicted $\operatorname{Pr}(\mathrm{D})>=.5$

True D defined as glp $!=0$

\begin{tabular}{lll} 
Sensitivity & $\operatorname{Pr}(+\mid \mathrm{D})$ & $84.80 \%$ \\
Specificity & $\operatorname{Pr}(-\mid \sim \mathrm{D})$ & $40.58 \%$ \\
Positive predictive value & $\operatorname{Pr}(\mathrm{D} \mid+)$ & $72.11 \%$ \\
Negative predictive value & $\operatorname{Pr}(\sim \mathrm{D} \mid-)$ & $59.57 \%$ \\
\hline- False + rate for true $\sim \mathrm{D}_{---}$ & $\operatorname{Pr}(+\mid \sim \mathrm{D})$ & $59.42 \%$ \\
False - rate for true D & $\operatorname{Pr}(-\mid \mathrm{D})$ & $15.20 \%$ \\
False + rate for classified + & $\operatorname{Pr}(\sim \mathrm{D} \mid+)$ & $27.89 \%$ \\
False - rate for classified - & $\operatorname{Pr}(\mathrm{D} \mid-)$ & $40.43 \%$
\end{tabular}

Correctly classified

69.07\%

La proporción de observaciones predichas correctamente es de $69.07 \%$.

\section{$\checkmark \quad$ La prueba de Hosmer-Lesmeshow}

La prueba de Hosmer-Lesmeshow, el cual es una prueba de bondad de ajuste donde:

H0: El modelo está bien ajustado

H1: Falta ajuste en el modelo

Number of observations $=388$

Number of groups $=10$

Hosmer-Lemeshow chi2(8) $=5.58$

Prob $>$ chi $2=0.6939$

Fuente: Elaboración de los autores. 
La probabilidad Prob > chi2 es mayor a 0.05, por tanto se acepta la hipótesis nula (H0).

\section{Presupuesto estimado para sustituir leña por Gas Licuado de Petróleo}

\begin{tabular}{|l|r|}
\hline Precio en dólares del cilindro (Envase 11.34 KG) & 35 \\
\hline Precio (Córdobas)* & 730.69 \\
\hline GLP (11.34 KG del hidrocarburo) $* *$ & 250 \\
\hline Mangueras & 130 \\
\hline Precio cocina sencilla (Córdobas) $* * * *$ & 500 \\
\hline Pago por transporte de equipo (Córdobas) & 50 \\
\hline TOTAL (Córdobas) & $\mathbf{1 6 6 0 . 6 9}$ \\
\hline INGRESO PROMEDIO FAMILIAR MENSUAL (Nominal) & $\mathbf{5 0 4 9 . 0 8}$ \\
\hline Peso de la inversión inicial sobre el ingreso promedio mensual & $\mathbf{3 3 \%}$ \\
\hline
\end{tabular}

*Tipo de cambio oficial 13/01/2010: 20.8768

** Precio máximo al consumidor- INE

*:* Se supuso un precio de una cocina sencilla de 4 quemadores sin horno.

Fuente: Distribuidora Tropigas “Martínez”. Teléfono: 505- 22603939

\section{Referencias bibliográficas}

Banco Central de Nicaragua. (2010). Anuario de Estadísticas Mensuales. Recuperado el 15 de enero de 2010 de http://www.bcn.gob.ni

Bluffstone, R. (1995). The effect of Labour Market Performance on Deforestation in Developing Countries under Open Access: An Example from rural Nepal. Journal of Environmental Economics and Management, (29), 42-63.

Briscoe, J. (1979). Energy use and social structure in a Bangladesh village. Population and Development Review, 5 (4), 615-641.

Dasgaputa, P. S. (1993). Poverty, Resources, and Fertility: the Household as a Reproductive Partnership. Environmental economics and natural resources management in developing countries. The World Bank.

FAO. (1997). Wood Energy, Women and Health. Regional Wood Energy Development Programme in Asia. FAO Report.

Filmer, D. \& Pritchett, L. (1996). Environmental Degradation and the Demand for Children: Searching for the Vicious Circle. Washington: World Bank.

French, D. (1981). The Energy studies unit and the Malawi Wood energy Project. Draft for FAO (Manuscrito no publicado).

Hamacher, G. S., Hyde, F. W. \& Joshee, B. R. (1993). Joint Production and Consumption in traditional Households: Fuelwood and Crop Residues in Two Districts in Nepal. The Journal of Development studies, 3 (1), 206-225. 
Hamacher, G. S., Hyde, F. W. \& Kanel, K. R. (1996). Household Fuelwood Demand and Supply in Nepal's Tarai and Mid-Hills: Choice Between and cash outlays and labour opportunity. World Development, 24(11), 1725-1736.

Instituto Nacional de Información de Desarrollo. (2005a). VIII Censo de Población y IV de Vivienda. Base de datos en soporte CD. Managua: INEC.

Instituto Nacional de Información de Desarrollo. (2005b). Encuesta de Medición del Nivel de Vida. Base de datos en soporte CD. Managua: INIDE.

Instituto Nicaragüense de Energía (2010). Recuperado el 10 de enero de 2010 de http://www. ine.gob.ni

Kumar, S. K. \& Hotchkiss, D. (1988). Consequences of deforestation for Women's time allocation, agricultural production, and Nutrition in Hill Areas of Nepal. Washington. International Food Policy Research Institute. Recuperado el 20 de enero de 2010 de http://ageconsearch.umn.edu/handle/42172

Leach, G. \& Gowen, M. (1987). Household Energy Handbook - An Interim Guide and Reference Manual. World Bank Technical Paper No. 67. Washington: The World Bank.

Martínez Cuéllar, M. (2003). La demanda por combustibles y el impacto de la contaminación al interior de los hogares sobre la salud: El caso de Guatemala. Tesis de Maestría publicada en Documento Cede 2003-2006. Universidad de los Andes. Colombia.

Meza González, C. (2009) Regulación del Mercado de Gas Licuado de Petróleo envasado en cilíndros de $11.34 \mathrm{~kg}$. Período 2000-2008. Conferencia brindada en noviembre 2009 en la Universidad Centroamericana para las asignaturas de Economía Empresarial y Regulación Económica, Managua, Nicaragua.

Ministerio de Energía y Minas. (2009). Encuesta para evaluar capacidad de pago por el servicio de energía eléctrica. Managua: MEM. Base de datos CD.

Moral, E. (2003). Modelos de elección discreta. Recuperado el 25 de enero de 2010 de http:// www.uam.es/personal_pdi/economicas/eva/pdf/logit.pdf

Multiconsult \& Cía. Ltda. (2005). Encuesta Nacional de Leña. Managua: Ministerio de Energía y Minas. (2005). Recuperado el 15 de diciembre de 2009 de http://www.mem.gob.ni/index. php?s $=51$.

Organización Mundial de la Salud. (2002). Informe Mundial de la Salud 2002. Ginebra: OMS

Pacundan, R. B. (1997). Exit from Lock-in to Energy Efficient Technologies: Household Cooking Stoves and Gender. Seminar of Gender and Technology.

Pitt, M. M. (1985). Equity, externalities and energy subsidies: The case of kerosene in Indonesia. Journal of Development Economics, 17 (3), 201-217.

Smith, K. R. (1993). Fuel combustion, air pollution exposure, and health: the situation in developing countries. Annual Review of Energy and Environment. 18, 529-566.

Smith, K. R., Bruce, N., Ballard, G. et al. (2000). Addressing the Impacts of household Energy and Indoor air Pollution on Health of the Poor - Implications for Policy Action and Intervention Measures. Recuperado el 10 de diciembre de 2009 de http://www.who.int/ mediacentre/events/H\&SD_Plaq_no9.pdf

Stevenson, G. (1989). The Production, Distribution, and Consumption of Fuelwood in Haiti. The Journal of Developing Areas (24, 59-76

Vargas, E. (2001). Acceso a los alimentos: factores que lo afectan. Recuperado el 20 de enero de 2010 de www.rlc.fao.org/iniciativa/cursos/Curso\%202006/Mod3/13e.ppt 